\begin{tabular}{ll}
\hline & Perioperative endocrine \\
& and electrolyte \\
Bart Chernow MDFACP & emergencies
\end{tabular}

Endocrine and electrolyte abnormalities are common perioperative problems. The purpose of this discussion is to review several of the more common hormonal and metabolic problems observed in the practice of anaesthesia. As opposed to the traditional approach of discussing aetiology, diagnosis, clinical manifestations and treatment of every endocrine and metabolic disorder, I will instead highlight those specific points of concern for anaesthetists.

\section{Thyroid dysfunction}

Hyperthyroidism is a common problem which is increasingly being treated with radioactive iodine and/or antithyroid medications. Surgery is less commonly performed as therapy for thyrotoxicosis, and with the use of betaadrenergic receptor antagonists, intraoperative "thyroid storm" is now very rare. Of greater concern to anaesthetists is the undiagnosed hyperthyroid patient who is undergoing anaesthesia in preparation for non-thyroidal surgery. These patients may develop tachycardia, marked increases in body temperature, diaphoresis and systolic hypertension (with a widened pulse pressure). The tachycardia may be either sinus tachycardia or atrial fibrillation with a rapid ventricular response. Surgery, trauma and infection are among the known precipitants of thyrotoxic crisis. If you are faced with a patient who develops tachycardia, systolic hypertension and fever, consider thyrotoxicosis in addition to the usual differential diagnosis for these intraoperative problems (including malignant hyperthermia drug reaction, inadequate anesthesia, etc.). The other hormonal abnormality that may present in this manner is phaeochromocytoma. For this reason, labetalol (a combined alpha and beta "blocker") is probably the initial drug of choice for alleviation of the sinus tachycardia/ hypertension. If propranolol were used and the patient had a norepinephrine-secreting "phaeo" then there might be the opportunity for unoppsded alpha-adrenergic receptor activation with severe hypertension. Labetalol will prevent this problem. One way to distinguish rapidly hyperthyroidism from "phaeo" is to look at the patient's eyes for evidence of proptosis and palpate the thyroid gland, looking for goitre. In elderly patients with hyperthyroidism, these physical findings may be absent. At the time that hyperthyroidism is suspected, a blood sample should be sent to the laboratory for serum thyroxine $\left(T_{4}\right)$, triiodothyronine $\left(\mathrm{T}_{3}\right)$ by radioimmunoassay and thyrotropin (TSH or thyroid stimulating hormone) determinations.

\section{Clinical points}

1 In the patient with tachycardia, systolic hypertension and fever, consider thyrotoxicosis.

2 Always consider thyrotoxicosis in a patient with atrial fibrillation with a rapid ventricular response.

3 Labetalol may be the drug of choice for treatment of sinus tachycardia and hypertension of undetermined aetiology.

Hypothyroidism is also a common disease. Anaesthetists should consider this diagnosis in operative patients who develop unexplained postoperative hypothermia, in those who appear to have diminished ability to metabolize medications, in patients with unexplained sinus bradycardia and in patients who have difficulty in weaning from mechanical ventilatory support. Patients with hypothyroidism may have impairments in shivering thermogenesis, metabolism of medications and ventilatory responses to hypoxia and hypercapnia. They may also be anaemic, hyponatraemic and have hypercholesterolaemia. The myopathy which often is a component of hypothyroidism may lead to increases in serum creatinine phosphokinase (CPK) concentrations. The diagnostic test of choice is measurement of the serum TSH concentration. In primary (thyroprivic) hypothyroidism, the serum TSH value is increased above $5 \mathrm{mlU} \cdot \mathrm{ml}^{-1}$. In critically ill patients, serum $T_{4}$ and $T_{3}$ determinations are often not helpful tests, due to illness-induced changes in these variables. Dopamine and/or glucocorticoids may inhibit TSH release from the pituitary gland.

\section{Clinical points}

1 Consider hypothyroidism in patients with hypothermia or bradycardia, "difficult to wean" patients and in those with unexplained increases in serum CPK concentrations.

Department of Anesthesia (Critical Care), Harvard Medical School, Massachusetts General Hospital, Boston, MA U.S.A. 
2 Serum TSH is the test of choice in order to determine if a patient has primary hypothyroidism.

3 Perioperative decreases in serum total $T_{4}$ and $T_{3}$ concentrations are common in critically ill adults and children.

\section{Adrenal cortical insufficiency}

The adrenal cortex releases cortisol (a glucocorticoid), aldosterone (a mineralocorticoid) and androgenic (sex) steroids. Acute illness is associated with increases in the circulating concentrations of cortisol and aldosterone and decreases in sex steroids.

Primary adrenal insufficiency (Addison's disease) is most often caused by auto-immune disease; however, infections, granulomatous diseases (e.g., tuberculosis), haemorrhage and very rarely metastasis may also cause this condition. Primary adrenal insufficiency (i.e., destruction of the adrenal cortex) is associated with hyperpigmentation. Secondary adrenal insufficiency (i.e., due to lack of adrenal gland stimulation because of hypothalamic-pituitary dysfunction) is not accompanied by hyperpigmentation. Chronic glucocorticoid administration often leads to hypothalamic - pituitary-adrenal cortical axis suppression with resultant secondary adrenal insufficiency if glucocorticoid therapy is withheld. Several case reports in the 1950 s underscored this point, by describing perioperative hypotension and death in a handful of steroid-dependent patients who were not given perioperative glucocorticoid "coverage." Although an initial recommendation of $300 \mathrm{mg}$ of hydrocortisone (100 $\mathrm{mg}$ preoperatively, $100 \mathrm{mg}$ intraoperatively and $100 \mathrm{mg}$ administered postoperatively) was used for virtually all steroid-dependent patients, recent clinical observations suggest a need for a change in this approach. The $300 \mathrm{mg}$ approach is costly and may lead to an increased frequency of impaired wound healing, infection and in some cases hypokalaemic metabolic alkalosis. Based upon clinical findings in humans undergoing different "grades" of surgical stress, I recommend that the amount of glucocorticoid coverage be based upon three variables: (1) the amount of glucocorticoids the patient has been receiving preoperatively, (2) the length of time the patient has been receiving steroids, and (3) the anticipated length and severity of surgery.

\section{Clinical points}

1 Primary adrenal insufficiency (but not secondary adrenal insufficiency) is characterized by hyperpigmentation.

2 Perioperative glucocorticoid coverage is important, but should be titrated according to the patient's steroid history and the anticipated length of the operation.

\section{Electrolyte considerations}

Entire books have been devoted to electrolyte abnormalities. Therefore, this brief essay cannot be encyclopaedic about this subject area. Instead, I will detail several clinical "pearls" that I hope are clinically useful to the provider of anaesthesia or intensive care services:

1 Potassium - Always interpret the serum potassium concentration in light of the patient's arterial blood pH. Acidaemic patients should be hyperkalaemic and alkalamic patients should be hypokalaemic. Therefore, hypokalaemia in the setting of acidaemia is of much greater importance than the same hypokalaemia in the setting of alkalaemia. Similarly, hyperkalaemia is more serious in the alkalaemic patient than a similar degree of hyperkalaemia in the setting of acidaemia. Remember that both insulin and beta-2-adrenergic receptor agonists augment cellular uptake of potassium.

2 Sodium - Serum sodium often reflects body water and changes in water metabolism. Therefore, hypernatraemia reflects water depletion - as we might see in a patient with diabetes insipidus (DI). Hyponatraemia reflects water excess - for example, in a patient with the syndrome of inappropriate antidiuretic hormone (SIADH). In postoperative ICU patients (without access to water by mouth), polyuria due to diabetes insipidus is always accompanied by an increase in the serum sodium concentrations. Therefore, in dealing with postoperative polyuria, performance of a serum sodium determination is a better way to exclude DI than the measurement of urine osmolality of electrolyte determinations.

3 Hypokalaemia and hypercalcaemia are the two electrolyte abnormalities associated with nephrogenic DI.

4 Calcium - Hypocalcaemia is a common electrolyte abnormality in postoperative ICU patients. The causes of hypocalcaemia in this patient population are multifactorial (including illness-associated changes in arterial $\mathrm{pH}$, free fatty acids, parathyroid hormone and administered salts such as citrate). Patients who develop ionized hypocalcaemia often have a worse outcome than those with normocalcaemia; however, no cause and effect relationship has been established. Experimental and clinical evidence support the concept that maintenance of a near normal circulating ionized calcium concentration may be efficacious; however, several experimental studies argue against aggressive calcium replacement therapy.

5 Magnesium-Hypomagnesaemia, like hypocalcaemia, is a common postoperative problem in ICU patients. The mechanisms underlying this problem are similar to those for hypocalcaemia. Endocrine and electrolyte problems are common in postoperative ICU patients. 
Clinicians should consider some of these special problems in their care of this patient population.

\section{References}

1 Sheps SG, Jiang NA, Klee GG, van Heerden JA. Recent developments in the diagnosis and treatment of pheochromocytoma. Mayo Clin Proc 1990; 65:

88-95.

2 Zaloga GP. Chernow B, Smallidge RC et al. A longitudinal evaluation of thyroid function in critically ill surgical patients. Ann Surg 1985; 201: 456-63.

3 Zwillich CW, Pierson DJ, Hofeldt FD et al. Ventilatory control in myxedema and hypothyroidism. N Engl J Med 1975; 292: 662-5.

4 Ladenson PW, Levin AA, Ridgway EC, Daniels GH. Complications of surgery in hypothyroid patients. Amer J Med 1984; 77: 261-6.

5 Chernow B, Alexander $R$, Smallridge $R C$ et al. Hormonal responses to graded surgical stress. Arch Intern Med 1987; 147: 1273-8.

6 Napolitano LM, Chernow B. Guidelines for corticosteroid use in anesthetic and surgical stress. Intern Anesth Clinics 1988; 26: 226-32.

7 Zaloga GP, Chernow B. Hypocalcemia in critical illness. JAMA 1986; 256: 1924-9.

8 Chernow B, Bamberger S. Stoiko M et al. Hypomagnesemia in patients in postoperative intensive care. Chest 1989; 95: 391-7. 


\section{Problèmes endocri- niens et électrolytiques d'urgence en anesthésie}

Les anomalies endocriniennes et électrolytiques sont des problèmes péri-opératoires courants. Le but de cette discussion est de revoir plusieurs des problèmes hormonaux et métaboliques les plus courants observés dans la pratique de l'anesthésie. Plutôt que d'utiliser une approche de discussion traditionnelle, je vais plutôt souligner les points spécifiques qui intéressent les anesthésistes.

\section{Dysfonction thyroidienne}

L'hyperthyroidie est un problème courant qui est de plus en plus traité avec de l'iode radioactif et/ou une médication anti-thyroïdienne. On utilise moins couramment la chirurgie pour la thérapie de la thyrotoxicose, et avec l'utilisation des antagonistes des récepteurs bêta, la tempête thyroïdienne per-opératoire est maintenant très rare. Le patient hyperthyroïdien non diagnostiqué qui est anesthésié pour une chirurgie non thyroïdienne est beaucoup plus inquiétant. Ces patients peuvent développer de la tachycardie, des augmentations importantes de température, de la diaphorèse et une hypertension systolique (avec une pression différentielle plus grande). La tachycardie peut être sinusale ou se manifester en fibrillation auriculaire avec une réponse ventriculaire rapide. $\mathrm{La}$ chirurgie, les traumatismes et les infections sont parmi les agents déclanchants de crise thyrotoxique. Si vous êtes en face d'un patient qui développe de la tachycardie, une hypertension systolique et une augmentation de température, il vous faut inclure la thyrotoxicose en plus des autres diagnostics différentiels pour ce genre de problème intraoperatoire (hyperthermie maligne, réaction aux médicaments, anesthésie inadéquate, etc.). L'autre problème hormonal qui peut se présenter de cette façon est le phéochromocytome. Pour cette raison, le labétalol (un bloqueur alpha et bêta combiné) est probablement la substance de premier choix pour diminuer la tachycardie sinusale et l'hypertension. Si le propanolol était utilisé et que le patient avait un phéochromocytome sécréteur de norépinéphrine, on pourrait se trouver en face d'une occasion d'activation non limitée des récepteurs alpha, avec tableau d'hypertension sévère. Le labétalol va prévenir ce problème. Il y a une façon de distinguer rapidement entre l'hyperthyroïdie et le phéochromocytome: il s'agit d'examiner les yeux du patient et de chercher la proptose, et de palper la glande thyroïde, en cherchant un goître. Chez les patients âgés avec hyperthyroïdie, ces signes physiques peuvent être absents. Au moment où l'hyperthyroïdie est suspectée, on devrait envoyer un échantillon de sang au laboratoire pour doser la thyroxine $\left(\mathrm{T}_{4}\right)$, la triiodothyronine $\left(\mathrm{T}_{3}\right)$ par épreuve radioimmunologique, ainsi que la thyrotropine (TSH).

\section{Conseils cliniques}

I Chez le patient avec tachycardie, hypertension systolique et fièvre, penser à la thyrotoxicose.

2 Toujours penser à la thyrotoxicose chez un patient avec une fibrillation auriculaire et réponse ventriculaire rapide.

3 Le labétalol peut être le médicament de choix pour le traitement de la tachycardie sinusale et de l'hypertension d'origine indéterminé.

L'hypothyroidie est aussi une maladie courante. Les anesthésistes devraient penser à ce diagnostic chez les patients opérés qui développent une hypothermie postopératoire d'origine inexpliquée, chez ceux qui semblent avoir une capacité diminuée de métaboliser les substances, chez les patients avec bradycardie sinusale inexpliquée et chez ceux qui ont des difficultés à être sevrés de la ventilation mécanique. Les patients avec hypothyroïdie peuvent avoir une diminution de la thermogénèse en réponse au frisson, du métabolisme des médicaments et de la réponse respiratoire à l'hypoxie et à l'hypercapnie. Il peuvent aussi être anémiques, hyponatrémiques, et être porteurs d'hypercholestérolénie. La myopathic qui souvent est une composante de l'hypothyroïdie, peut amener des augmentation de la créatine phospokinase sérique. L'épreuve diagnostique de premier choix est la mesure de la concentration sérique de TSH. Dans l'hypothyroïdie primaire, la valeur de TSH sérique est augmenté $>5$ $\mathrm{mIU} \cdot \mathrm{ml}^{-1}$. Chez les patients très malades, la $T_{4}$ et la $T_{3}$ sériques sont souvent des épreuves peu utiles, à cause des variations apportées par la maladie. La dopamine et/ou les glucocorticoïdes peuvent inhiber la libération de TSH par l'hypophyse.

\section{Conseils cliniques}

1 Penser à l'hypothyroïdie chez les patients avec hypothermie ou bradycardie, difficiles à sevrer, et chez ceux 
où l'on retrouve des augmentations inexpliquées de la concentration de CPK.

2 La TSH sérique est l'élément de choix à analyser si le patient a une hypothyroïdie primaire.

3 Les diminutions péri-opératoires de $T_{3}$ et $T_{4}$ sériques sont courantes chez les adultes et les enfants sévèrement malades.

\section{Insuffisance adréno-corticale}

Le cortex surrénalien libère le cortisol (un glucocorticoïde), l'aldostérone (un minéralocorticoöde) et des stéroïdes androgéniques. La maladie aigüe est associée à des augmentations de cortisol et d'aldostérone et à une diminution des hormones sexuelles. L'insuffisance surrénalienne primaire (maladie d'Addison) est le plus souvent causée par une maladie auto-immune ; cependant, des infections, des maladies granulomateuses (e.g., tuberculose), l'hémorragie et très rarement des métastases, peuvent aussi amener cette condition. L'insuffisance surrénalienne primaire (i.e., destruction du cortex surrénalien) s'associe à de l'hyperpigmentation. L'insuffisance surrénalienne secondaire (due au manque de stimulation de la surrénale par suite d'une dysfonction hypothalamo-hypophysaire) ne s'accompagne pas d'hyperpigmentation. L'administration chronique de corticoïdes va souvent conduire à une suppression de l'axe entre l'hypothalamus, l'hypophyse et le cortex surrénalien, avec comme résultat une insuffisance surrénalienne secondaire, lorsque l'on interrompt la thérapie aux glucocorticoïdes. Plusieurs cas rapportés dans les années 1950 soulignant ce point, avec description d'hypotension périopératoire et décès, chez un petit nombre de patients cortico-dépendants qui n'avaient pas reçu de couverture péri-opératoire en glucocorticoïdes. Même si une recommandation initiale de $300 \mathrm{mg}$ d'hydrocortisone $(100 \mathrm{mg}$ en pré-opératoire, $100 \mathrm{mg}$ pendant la chirurgie, $100 \mathrm{mg}$ administré après l'intervention) est d'usage courant pour à peu près tous les patients cortico-dépendants, des observations cliniques récentes suggèrent un changement dans cette approche. L'approche à $300 \mathrm{mg}$ est coûteuse et peut conduire à une augmentation de fréquence de mauvaise guérison, d'infection et dans certains cas à une alkalose métabolique hypo-kaliémique. En se basant sur des trouvailles cliniques chez les personnes soumises à différents niveaux de stress chirurgical, je recommande que les quantités de glucocorticoïdes de couverture soient établies sur trois variables. 1) La quantité de glucocorticoïdes que le patient recevait avant l'opération; 2) la durée de la thérapie aux stéroïdes; et 3 ) la durée et la sévérité anticipées de la chirurgie.

\section{Conseils cliniques}

1 L'insuffisance surrénalienne primaire (pas l'insuffi- sance secondaire) se caractérise par de l'hyperpigmentation.

2 La couverture péri-opératoire avec glucocorticoïdes est importante, mais devrait être ajustée selon l'histoire de corticothérapie du patient et la durée anticipée de la chirurgie.

\section{Considérations sur les électrolytes}

Des livres entiers ont traité des anomalies électrolytiques. Pour cette raison, cette courte présentation ne peut pas être encyclopédique sur ce sujet. Je vais plutôt parler en détail de quelques «perles » cliniques qui j'espère seront utiles pour l'anesthésiste et l'intensiviste:

1 Potassium - il faut toujours interpréter la kaliémie en fonction du $\mathrm{pH}$ artériel du patient. Les patients en acidose devraient être hyperkaliémiques et les patients alkalêmiques devraient être hypokaliémiques. Dès lors, l'hypokaliémie en présence d'une acidémie a beaucoup plus d'importance que la même hypokaliémie dans un contexte d'alkalémie. De la même façon, l'hyperkaliémie est plus sérieuse chez le patient alkalémique qu'un degré semblable d'hyperkaliémie dans un contexte d'acidémie. Rappelez-vous que l'insuline et les agonistes des récepteurs bêta-2 vont augmenter la captation cellulaire de potassium.

2 Sodium - la natrémie va souvent être le reflet de l'eau corporelle et des changements du métabolisme de l'eau. Dès lors, l'hypernatrémie reflète une perte d'eau, comme on peut le constater chez un patient avec diabète insipide. Hyponatrémie signifie quantité excessive d'eau, par exemple chez le patient avec syndrome de sécrétion inappropriée d'hormone anti-diurétique (SIADH). Chez les patients dans les unités de soins intensifs (qui n'ont pas accès à de l'eau par la bouche) la polyurie dûe au diabète insipide s'accompagne toujours d'une augmentation de la natrémie. Dès lors, lorsque nous avons à nous occuper d'une polyurie en postopératoire, la quantification de la natrémie est un meilleur moyen d'exclure le diabète insipide que la mesure de l'osmolarité urinaire.

3 L'hypokaliémie et l'hyperkalcémie - ce sont deux anomalies électrolytiques associées au diabète insipide néphrogénique.

4 Calcium - l'hypocalcémie est une anomalie courante en période post-opératoire aux soins intensifs. Les causes d'hypocalcémie dans ce groupe de patients sont plurifactorielles (comprennent les changements de $\mathrm{pH}$ artériel associés à la maladie, les acides gras libres, la parathormone et les sels administrés tels que le citrate). Les patients qui voient chuter leur calcium ionisé ont souvent un pronostic moins bon que ceux qui conservent un calcium normal. Cependant, on n'a pu établir de relation cause-effet à ce niveau. Les données expé- 
rimentales et cliniques appuient le concept de l'efficacité du maintien d'une concentration de calcium ionisé à des niveaux à peu près normaux ; cependant, plusieurs études expérimentales mettent en garde contre une thérapie agressive de remplacement du calcium.

5 Magnésium - l'hypomagnésémie, comme l'hypocalcémie, est un problème post-opératoire courant chez les patients des unité de soins intensifs. Les mécanismes sous-jacents à ce problème sont semblables à ceux qui surgissent dans l'hypocalcémie.

Les problèmes électrolytiques et endocriniens sont courants chez les patients admis dans les unités de soins intensifs. Les cliniciens devraient porter attention à ces problèmes spéciaux lorsqu'ils ont la charge de cette population de patients.

\section{Références}

(Voir page Slxxiii) 